\title{
Settlement of a foundation slab, non-uniform in depth
}

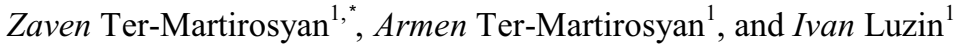 \\ ${ }^{1}$ Moscow state university of civil engineering, Yaroslavskoye shosse, 26, Moscow, Russia, 129337
}

\begin{abstract}
The paper describes the formulation and solution of problems for the quantitative evaluation of settlements and bearing capacity of rectangular and circular foundation slabs constructed on the base with deformation $(K, G$ or $E, v)$ properties of continuously varying heterogeneity in the depth due to the conditions of its formation. It is shown that the inhomogeneity of the deformation properties of the foundation soils over depths has a significant effect on the formation of additional stress and strain states under the influence of a uniformly distributed external load over the area of a rectangle and circle, where, with growth of loading area $(A)$ relative settlement $(S / \sqrt{A})$ depends nonlinearly on $\sqrt{A}$ and decreases with growth of $\sqrt{A}$ up to two or more times. As a computational model for the soil base, the paper considers the nonlinear geomechanical Klein model, according to which the stress-strain modulus of soils increases with depth according to the law of a power function of the form: $E(z)=E_{1} z^{n}$, where $n \leq 1$. The solution of the SSC problems for an inhomogeneous ground half-space under the influence of a local load was obtained by an analytical method using the Mathcad software complex on the basis of the Boussinesq-Frohlich concentrated force problem. The results of the solution are presented in tables and distribution diagrams $\sigma_{z}$ and $S(z)$, as well as $S=f_{2}(\sqrt{A}, n)$.
\end{abstract}

\section{Introduction}

Experience in the design, construction and operation of high-rise buildings and heavy energy facilities on large-scale foundation slabs with an area of more than $1000 \mathrm{~m}^{2}$ has shown that the design settlement calculated from the homogeneous half-space model significantly exceeds the actual settlements (more than 2 times) and that the scale factor of the slab foundation requires consideration.

The heterogeneity of the foundation $(E(z))$ has a significant effect on the nature of the formation of an additional stress-strain condition (SSC) under the influence of an external load transmitted through a large-scale slab foundation.

\footnotetext{
* Corresponding author: gic-mgsu@mail.ru
} 
The present paper describes the formulation and solution of problems on the quantitative estimation of the SSC of an inhomogeneous foundation under the influence of an external load uniformly distributed over the area of a rectangle and a circle, and for different proportions of length and width $(l / b, b=2 a)$, as well as $\left(l \cdot b\right.$ or $\left.\pi R^{2}\right)$, including dependencies $S(P)=f(\sqrt{A}, n)$.

As a computational geomechanical model, a hypothetical Klein foundation model was adopted, in which the stress-stain modulus increases continuously with depth in accordance with the power law

$$
E(z)=E_{1} \cdot z^{n},(n \leq 1)
$$

where $E_{1}$ - stress-strain modulus at a depth of $1 \mathrm{~m}, n$ - heterogeneity parameter.

To determine additional SSC of an inhomogeneous foundation from an external load, the well-known solution of the Boussinesq-Frohlich problem of force applied to an inhomogeneous half-space is considered as the initial one:

$$
\sigma_{z}=\frac{v \cdot P}{2 \pi} \cdot \frac{z^{v}}{R^{v+2}}
$$

where $v$ - stress concentration factor $(v \geq 3)$. At $v=3$, the solution corresponds to a homogeneous half-space.

\section{The SSC of a reconsolidated ground half-space under the influence of concentrated force (the Boussinesq and Frohlich- Klein problem)}

It is known [1-2] that under the action of a concentrated force on a ground-half-space, homogeneous in depth and along strike, the stress components and surface settlements are determined by the Boussinesq formulas:

$$
\begin{gathered}
\sigma_{z}=\frac{3 P}{2 \pi} \cdot \frac{z^{3}}{R^{5}}, \\
\sigma_{x}=\frac{3 P}{\pi}\left\{\frac{z x^{2}}{R^{5}}+\frac{1-2 v}{3}\left[\frac{R^{2}-R \cdot z-z^{2}}{R^{3}(R+z)}-\frac{x^{2}(2 R+z)}{R^{3}(R+z)^{2}}\right]\right\}, \\
\sigma_{y}=\frac{3 P}{\pi}\left\{\frac{z y^{2}}{R^{5}}+\frac{1-2 v}{3}\left[\frac{R^{2}-R \cdot z-z^{2}}{R^{3}(R+z)}-\frac{y^{2}(2 R+z)}{R^{3}(R+z)^{2}}\right]\right\}, \\
\sigma_{m}=\frac{P}{\pi}(1+v) \frac{z}{R^{3}}, \\
S=\frac{P(1+v)}{2 \pi E}\left[\frac{z^{2}}{R^{3}}+\frac{2(1-v)}{R}\right],
\end{gathered}
$$

where $R=x^{2}+y^{2}+z^{2}, v$ - Poisson's ratio, $P$ - concentrated force.

These dependences do not take into account the intrinsic weight of the soils and the initial stress state, which can cause the inhomogeneity of the initial SSC of the array in depth, and, consequently, the inhomogeneity in deformation properties. Given this circumstance, Frohlich [3] proposed the formula for $\sigma_{z}$ of Boussinesq (3) to introduce the stress concentration parameter $v$ to better match the results of experimental studies, i.e. suggested to present the dependency $\sigma_{z}(P, x, y, z)$ if the form of (2). Klein has showed [4] that dependence (2) satisfies the equilibrium conditions for any value, and the conditions of deformation continuity only at the following values: 


$$
\begin{aligned}
& v=n+3 \text { at } \mu_{0}=\frac{1}{2+n} \\
& v=n+2 \text { at } \mu_{0}=\frac{1}{1+n}
\end{aligned}
$$

where $\mu_{0}$ - coefficient of lateral expansion.

In addition, Klein has considered the problem of stress distributions $\sigma_{z}$ under the center of a rectangular and circular shape of the areas and compiled the table of corresponding coefficients $K_{0}(b / z)$ and $K_{0}(a / z)$. For the case $v=3$, Love obtained formulas for determining the stresses along the vertical lines passing under the center and at the angle of the rectangle [5]. According to this decision, the tables were compiled listed in SP 22.13330.2011. Below we consider the problem of determining stresses $\sigma_{z}$ on any vertical within and outside the rectangle at $v>3$, i.e. at $v=5$.

\section{SSC of an inhomogeneous ground half-space under the influence of a uniformly distributed load over the area of a rectangle for the case of an inhomogeneous half-space}

For the reliable In the case of a uniformly distributed load with intensity $p=P / A$ over the area of the rectangle $(2 b \geq 2 a)$, stresses $\sigma_{z p}(z)$ on any vertical passing through the points $M(x, y)$ on the surface, on the basis of (1) can be determined by the formula:

$$
\sigma_{z p}=\frac{v p z^{v}}{2 \pi} \int_{-a-b}^{+a+b} \frac{d \xi d \eta}{\left.\left[(x-\xi)^{2}+(y-\eta)^{2}\right)+z^{2}\right]^{\frac{v+2}{2}}}
$$

Comparative evaluation $\sigma_{z p}$ at $v=3$ and $v=5$ is shown in Fig. 1 with an indication of the lower boundary of the compressible thickness, determined from the condition $\sigma_{z p}=0,5 \cdot \sigma_{z g}$.

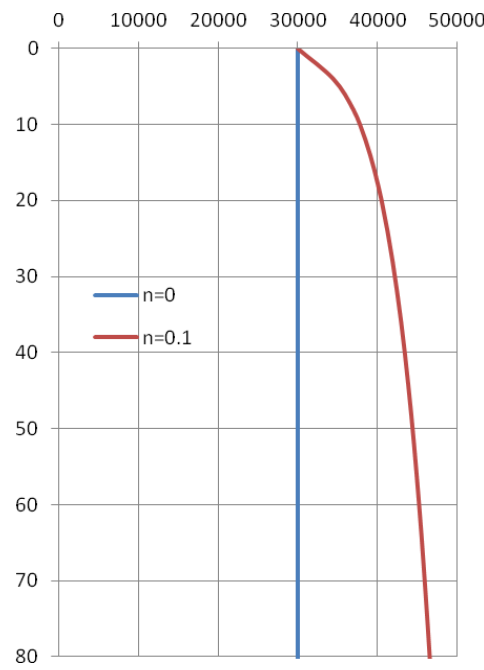

(a)

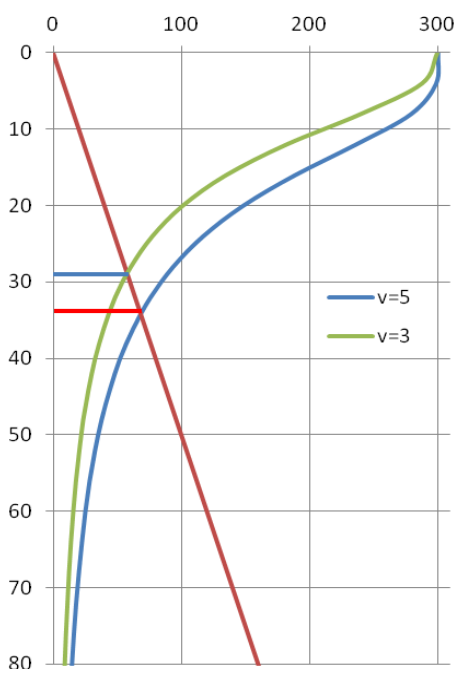

(b)

Fig. 1. Distribution diagrams $E(z)$ (a), made from (1) at $n=0$ (blue line) and $n=0,1$ (red line), and $\sigma_{z p}(z)$ (b), made from (5) at $v=3$ (green line) and $v=5$ (blue line) with an indication of the corresponding lower limits of the compressible thickness at $\sigma_{z g}=0,5 \cdot \sigma_{z p}$ 
Settlements at any point of the surface of an inhomogeneous half-space, $M(x, y)$ on the basis of (8) can be determined by integrating the relative deformation $\varepsilon_{z}(x, y)$, i.e.

$$
S(x, y)=\int_{0}^{h} \varepsilon(z) \cong 0,8 \int \frac{\sigma_{z}(z)}{E(z)} d z
$$

where $E(z)$ is determined by $(1), \sigma_{z}(z)$ - by (8), $h$ - power of compressible thickness. Putting (7) into (6), we obtain

$$
S(x, y)=\frac{v p}{2 \pi E_{1}} \int_{0}^{h} z^{v} \int_{-a}^{+a+b} \int_{-b}^{\frac{d \xi d z}{\left[(x-\xi)^{2}+(y-\eta)^{2}+z^{2}\right]}} d z
$$

where $h$ - lower boundary of the compressible thickness. Integration of this expression with the help of the Mathcad PC showed that the settlement in the center of the loaded area of the rectangle is 4 times larger than at the corner point, which coincides with the solution of a homogeneous half-space. The advantage of this method of solving the Love problem lies in the fact that it allows determining the settlement of any point of the surface both of as heterogeneous $(v>3)$ and homogeneous $(v=3)$ ground half-space within $\underline{\text { and }}$ a loading area and to build settlement isolines of the surface. This allows determining the bedding value under a rectangular foundation $C(x, y)=\sigma_{z}(x, y) / S(x, y)$.

\section{Influence of the loading area of a rectangular foundation on the settlement value of an inhomogeneous half-space}

To determine the deformation characteristics Expression (10) makes it possible to quantify the effect of the loading area $A=l \cdot b$ on the value of relative average settlement $\bar{S}$, where $\bar{S}=\left(S_{0}+S_{y 2}\right) / 2$, at any values of the inhomogeneity index $n(1)$, at any aspect ratio of a rectangular loading area $(l: b)$ and for a given distributed load $p$ for $v=3 ; 4$ and 5. Given that $S_{0}=4 S_{c}$, we can write $\bar{S}=5 S_{0} / 8$. Calculations performed for different values $\sqrt{A}=10,25,50,75,100$ and at the same load values $p$ have shown a nonlinear dependence between the average settlement $\bar{S}$ and value of $\sqrt{A}$ in the form

$$
\bar{S}_{i}=S_{10}-\alpha(n) \ln \left(A_{i} / A_{10}\right)
$$

where $S_{10}$ - settlement of a foundation with area of $10 \mathrm{~m}^{2}, \alpha(n)$ - coefficient depending on the degree of heterogeneity of the foundation $n, A_{i}$ - area of $i$-th foundation, wherein $A_{i}>A_{10}$. By constructing dependencies $\bar{S}-\sqrt{A}$ for values $n=0 ; 0,25 ; 0,5$ and 1,0 , we obtain a family of lines (Figure 2). Dependence of average settlement $\bar{S}$ on the degree of heterogeneity of the foundation $n$ can also be obtained by comparing the foundation settlements with fixed values of the foundation area ( $A=a \cdot b=c o n s t)$, stress concentration factor $v$ and the intensity of the applied load $p$ given (8) for different values of $n$. Analysis of these dependences at $v=5$ have shown that under identical loads $p_{1}=p_{2}=p$ at $v=5$ we can obtain a relation of the form

$$
\frac{\bar{S}_{1}}{\bar{S}_{2}}=\left(\frac{\sqrt{A_{1}}}{\sqrt{A_{2}}}\right)^{1,3-n}
$$




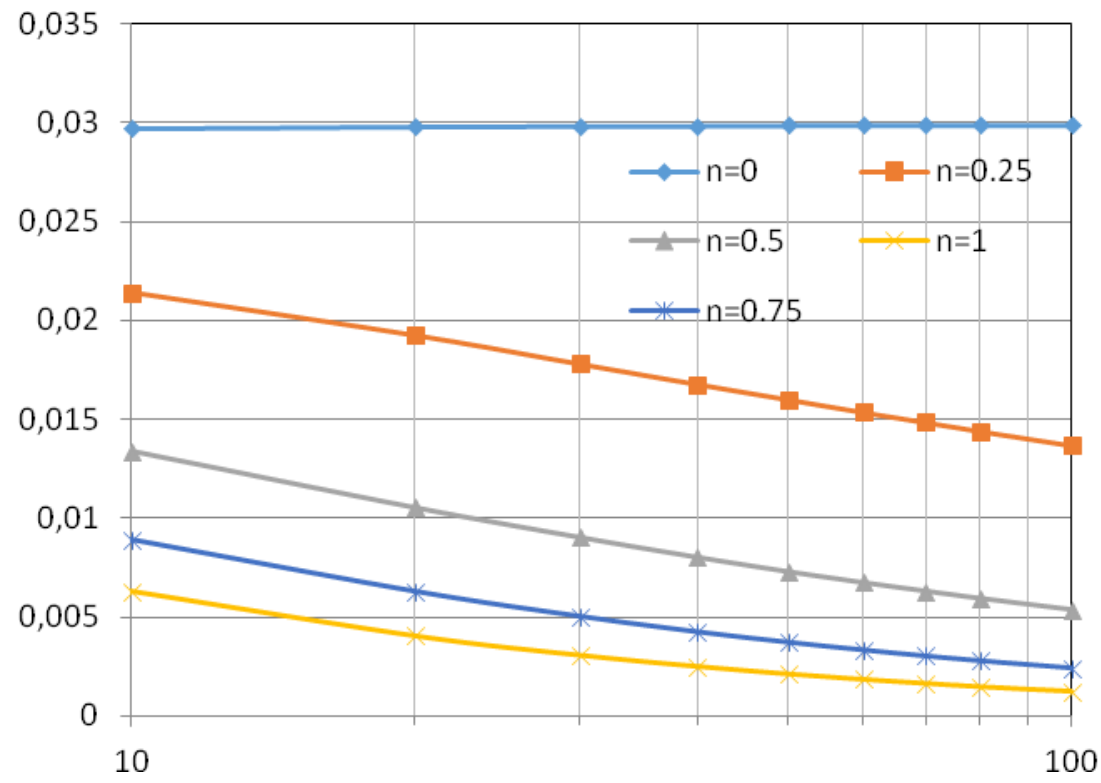

Fig. 2. Dependence of relative settlement $(S / \sqrt{A})$ on $\sqrt{A}$ on a foundation, homogeneous $(n=0)$ and a heterogeneous $(n=0,25 ; 0,5 ; 0,75$ and 1$)$ in depth, on a semilogarithmic scale

\section{Determination of the settlement of a reconsolidated heterogeneous foundation under a distributed load along the area of the circle}

Dependence of average settlement $\bar{S}$ on the degree of heterogeneity of the foundation $n$ can also be obtained by comparing the foundation settlements with fixed It is known that the SSC of a homogeneous foundation $(n=0, v=3)$ under the influence of the distributed load with intensity $p[\kappa \Pi a]$ over the area of a circle of radius $r=a$ is determined by the formulas

$$
\begin{aligned}
& \sigma_{z p}=p\left[1-\frac{z^{3}}{\left(a^{2}+z^{2}\right)^{3 / 2}}\right] \\
& S(r=0)=\frac{2\left(1-v^{2}\right) a p}{E}
\end{aligned}
$$

For the general case, when $n \leq 1, v>0,3$, the stress distribution is $\sigma_{z}$ on any vertical within and outside the loaded area of the circle (Figure 3) can be determined given (6) by the formula:

$$
\sigma_{z}=\frac{v \cdot z^{v} p}{\pi} \int_{0}^{a} \int_{0}^{2 \pi} \frac{\rho d \rho d \theta}{\left(\rho^{2}+b^{2}+z^{2}-2 b p \cdot \cos \theta\right)^{\frac{v+2}{2}}}
$$

where at $0<b<a$, the point is within the area of the circle, and at $b>a$-outside. At $v=5$ and $b=0$ we obtain $\sigma_{z}$ for the center point

$$
\sigma_{z}=5 \cdot z^{5} p \int_{0}^{a} \frac{\rho d s}{\left(\rho^{2}+z^{2}\right)^{3,5}}
$$




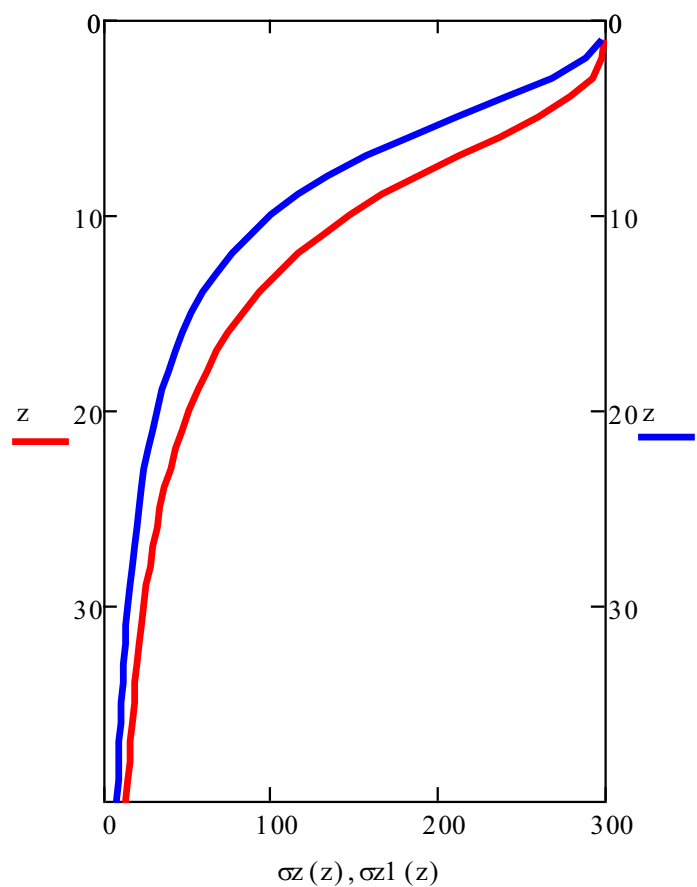

Fig. 3. Distribution of vertical stresses $\sigma_{z}$ at $v=3$ (blue line) and $v=5$ (red line) by depth calculated by the formula (13)

The integral over (14) is known, i.e.

$$
\sigma_{z}=\frac{5 \cdot z^{5} p}{3}\left[\frac{1}{z^{3}}-\frac{1}{\left(\sqrt{\left.a^{2}+z^{2}\right)^{3}}\right.}\right]
$$

To determine the settlement at the point $r=0$, we can use the expression (8); then, taking (15) into account, we obtain

$$
S(0)=\frac{4 p}{3 E_{1}} \int_{0}^{z} \frac{z^{5}}{z^{n}}\left[\frac{1}{z^{3}}-\frac{1}{\left(a^{2}+z^{2}\right)^{3 / 2}}\right] d z
$$

Calculations from (16) show that $S(0)$ depends nonlinearly on the radius of the circle $r=a$, and with increasing radius, the settlement $S(0)$ decreases; for the case of a homogeneous $n=0$ and heterogeneous $n=0,5$ of the foundation, the settlements at $a=5 \mathrm{~m}$ differ by a factor of 2 .

To determine the settlements of other points on the surface of an inhomogeneous foundation, it is necessary to integrate (13) at $b>a$. Calculations show that the deflection of the surface of an inhomogeneous foundation under uniform load along the area of the circle has the form (Fig.). Comparing this curve with Zaretsky influence curve [6], we see that they coincide qualitatively. Comparing the settlements of the surface of two circular foundations with area of $A_{1}$ and $A_{2}$ for an inhomogeneous foundation, all other conditions being equal, we see that the dependence $S_{1} / S_{2}$ has the form:

$$
\frac{S_{1}}{S_{2}}=\frac{\sqrt{A_{1}}}{\sqrt{A_{2}}}
$$

It should be noted that Yu.K. Zaretsky hasn't considered when solving the problem of drafting a circular foundation on a surface, non-homogeneous by Klein. Only the influence coefficient was used $K(r)$, which to some extent is an approximation. The results of this 
solution in the form of a function $S_{1} / 2=f\left(A_{1} / A_{2}, n\right)$ were included in the SNiP 2.02.02 85 . However, stress distribution $\sigma_{z}$ by depth in calculation of the settlement by the method of layerwise summation was determined for a homogeneous foundation, which is also a certain approximation.

The results of long-term observations of settlements of the foundations of buildings and structures show that the design settlement often exceeds the actual one by a factor of 2-5 [7]. Consequently, based on the above solution, it is possible to introduce an averaged correction coefficient that increases the stress-stain modulus in calculating the sediment of the foundations of buildings and structures.

\section{Conclusion}

1. The SSC of a foundation, heterogeneous in depth, $\left(E(z)=E_{1} z^{n}, n<1\right)$ is substantially different from the SSC of a homogeneous foundation $(n=0)$, including foundations with an area of more than $1000 \mathrm{~m}^{2}$.

2. When designing large-scale foundations of high-rise buildings and heavy power plants, it is necessary to take into account the variability of the soil deformation modulus of the foundation according to Klein.

3. The heterogeneity of deformation properties of soils in depth $E(z)$ may be due to the history of formation of the earth cover, as well as the variability of the initial stress state $E(z)=f\left(\sigma_{z g}\right)$.

\section{Acknowledgements}

This study was performed with the financial support of the RF Ministry of Education and Science, grant №7.3225.2017/ Project Part.

All tests were carried out using research equipment of Head Regional Collective Research Centre of Moscow State University of Civil Engineering.

\section{References}

1. Y. Boussinesq. Application des potentiels à l'étude de l'équilibre et du mouvement des solides élastiques, Paris, 1885

2. V.A. Florin. Osnovy mekhaniki gruntov. Moscow, Gosstroyizdat, T.I, (1961).

3. O.K. Frohlich. Raspredeleniye davleniy v grunte. Moscow, Stroyizdat, (1933).

4. G. K. Klein. Uchet neodnorodnosti razryvnykh deformatsiy i drugikh mekhanicheskikh svoystv gruntov pri raschete sooruzheniy na sploshnom osnovanii. MCEI collected papers. 14, pp. 85-88, (1956).

5. A. Love. Matematicheskaya teoriya uprugosti. ONTI, (1935).

6. Yu.K. Zaretsky, M.Yu Garitselov. Glubinnoye uplotneniye gruntov udarnymi nagruzkami. Moscow, Energoizdat, (1989).

7. Z.G. Ter-Martirosyan. Mekhanika gruntov. Moscow. ASV, (2009).

8. M. Mitew-Czajewska, ACE 62(4), 73 (2016) 